This item was submitted to Loughborough's Institutional Repository (https://dspace.lboro.ac.uk/) by the author and is made available under the following Creative Commons Licence conditions.

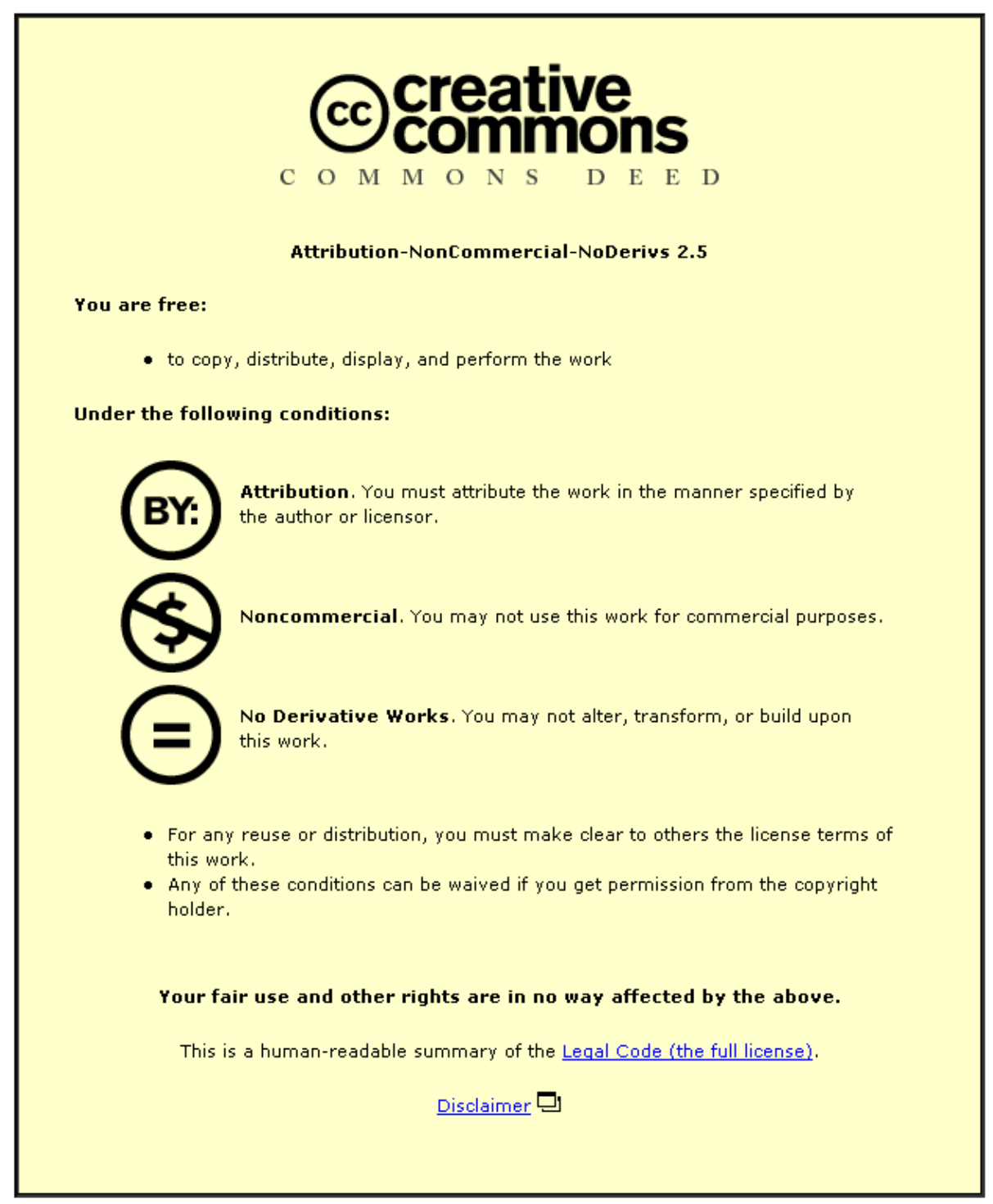

For the full text of this licence, please go to: http://creativecommons.org/licenses/by-nc-nd/2.5/ 


\title{
Strong Wiggler Field Assisted Amplification in a Second-Harmonic Waveguide Free Electron Laser
}

\author{
Xiehe Zhong, Student Member, IEEE, and Michael G. Kong, Senior Member, IEEE
}

\begin{abstract}
As a technique to reduce the size of compact waveguide free electron lasers (FELs) operated from microwave to the far infrared, a longitudinal interaction mechanism was recently proposed to operate waveguide FELs at the second harmonic. With a gain formulation based on Madey's theorem in the limit of small wiggler field, it was shown analytically that second harmonic waveguide FELs can reduce significantly the electron energy required for radiation at a given frequency. As it is advantageous to operate second harmonic waveguide FELs with strong wiggler field, Madey's theorem is used here to reformulate their interaction gain for strong wiggler fields up to $a_{w}^{2} / \gamma_{0}^{2} \beta_{z 0}^{2}=1$ with the axial electron velocity Taylor expanded to the eighth order of the wiggler field. Given that Madey's theorem has not been established for second harmonic waveguide FELs, their interaction gain is also formulated independently by solving their pendulum equation without recourse to Madey's theorem. These two gain formulas are not analytically identical, but numerically they lead to an excellent agreement over a wide range of system parameters, thus confirming the applicability of Madey's theorem. The interaction analyses presented form a thorough and detailed description of second harmonic waveguide FELs in the small-signal regime and for wiggler field that is both practical and beneficial.
\end{abstract}

Index Terms-Free electron lasers, harmonic generation, masers.

\section{INTRODUCTION}

C OMPACT waveguide free electron lasers (FELs) driven by low current electron beams have recently attracted much interest, largely because they offer a cost-effective means to deliver coherent radiation in the spectrum from microwave to the far infrared [1]-[9]. Aimed as a laboratory instrument rather than a national user facility [1], their output power is modest, up to a few kilowatts, but nevertheless adequate for many applications in basic research, medicine, and industry. Adding to the cost saving already achieved from employing low current electron beams, further system simplification and size reduction are now sought through improvement of their output power at a given beam current. This approach has led to the conception of many gain and efficiency enhancement techniques such as the employment of prebunched electron beams [6], [7] and the waveguide optical klystron arrangement [10].

Parallel to the efforts to improve the output power at a given electron beam current, an equally important issue in the development of the compact waveguide FEL technology is to reduce

Manuscript received December 4, 2000; revised December 13, 2001.

The authors are with the Department of Electronic and Electrical Engineering, Loughborough University, Leicestershire LE11 3TU, U.K. (e-mail: m.g.kong@lboro.ac.uk).

Publisher Item Identifier S 0093-3813(02)04654-4. the minimum electron energy needed to generate strong radiation at a given frequency [4], [11]-[13]. FEL operations at reduced electron energy not only permit the use of smaller and less expensive power supplies, but also increase the interaction gain indirectly through its inverse proportion to $\gamma^{\tilde{5}} \beta^{5}$ [3], [4]. Recently, an electron energy reduction technique was considered by exploiting the electron interaction with the longitudinal electric field component $E_{z}$ of TM modes in a cylindrical waveguide [14]. In such a beam-wave interaction configuration, electrons acquire first in the wiggler field a rapid longitudinal velocity oscillation twice as fast as their transverse velocity oscillation. Hence, their subsequent interaction with the $E_{z}$ component of TM modes may be considered as in an effective wiggler having a period half as much as that of the actual wiggler. For this reason, these free electron lasers are referred to as second harmonic waveguide FELs [14]. It was shown analytically that the electron energy required for radiation at a given frequency may be reduced significantly in second harmonic waveguide FELs from that needed in comparable conventional waveguide FELs where the transverse electron velocity couples with the transverse electric field component of TE modes in a rectangular waveguide [14], [15]. Second harmonic waveguide FELs are also different from conventional waveguide FELs in that they have a nonresonant electron velocity component that couples with the resonant velocity component to contribute significantly to the interaction gain [15]. This unique feature may be exploited to enhance the beam-wave interaction and to control the radiation spectrum.

Analytical treatments of second harmonic waveguide FELs have so far been based on the employment of Madey's theorem in the limit of small wiggler field [14], [15]. Compared to conventional waveguide FELs however, second harmonic waveguide FELs have a much stronger dependence on the wiggler field with their small-signal gain proportional to $B_{w}^{4}$ [15]. It is, therefore, preferable to operate second harmonic waveguide FELs with a strong wiggler field in order to enhance the interaction gain at a given beam current. As a result, our previous gain formulation developed in the limit of small wiggler field needs to be reassessed and, if necessary, extended. It is conceivable that in a sufficiently large wiggler field the oscillatory component of the axial electron velocity is no longer in a linear proportion to $B_{w}^{2}$ and in general the electron trajectory becomes a significantly nonlinear function of the wiggler field. This complexity in electron trajectory needs to be taken into account in any gain formulation of second harmonic waveguide FELs having a strong wiggler. In Section II, the nonlinear dependence of electron trajectory upon the wiggler field is considered with a Taylor expansion of the axial electron velocity to the eighth order of the wiggler field. With the electron velocity 
formulated, Madey's theorem is employed to reformulate the small-signal gain of second harmonic waveguide FELs. Comparison with the interaction gain obtained previously in the limit of small wiggler field [15] will be presented and relevant implications discussed.

It is important to note that the applicability of Madey's theorem to second harmonic waveguide FELs has not been established so far, although it is known to be applicable to conventional waveguide FELs [3], [10], [16]. Compared to their conventional counterparts, second harmonic waveguide FELs have a much more complex beam-wave interaction having the additional contribution of a nonresonant electron velocity component and its strong coupling with the usual resonant velocity component [15]. Consequently, it remains unclear whether these significantly new features of the beam-wave interaction make second harmonic waveguide FELs fundamentally different from conventional waveguide FELs, and thus compromise the validity of Madey's theorem. To address this issue, we derive in Section III a pendulum equation for second harmonic waveguide FELs and then employ perturbation theory to formulate the small-signal interaction gain without recourse to Madey's theorem. It will be shown that the gain formulation obtained from Madey's theorem and perturbation theory are not analytically identical though very similar. To illustrate their difference and similarities, numerical comparisons will be presented.

The two gain formulations developed with and without Madey's theorem provide a thorough assessment of the beam-wave interaction in second harmonic waveguide FELs in the small-signal regime and for a parametric range of wiggler field that is both practical and beneficial. Numerical examples presented and conclusions drawn are used to aid an enhanced insight into the beam-wave interaction mechanism in second harmonic waveguide FELs. These are summarized in Section IV.

\section{GAin Formulation Using MADEY's THEOREM}

Different from conventional waveguide FELs, second harmonic waveguide FELs generate strong radiation in TM modes in a cylindrical waveguide. Wiggler magnets used for these new FELs are usually planar and in the one-dimensional (1-D) limit their magnetic field may be approximated with the following on-axis expression:

$$
B_{w}=\hat{x} B_{w 0} \cos k_{w} z
$$

where $k_{w}=2 \pi / \lambda_{w}$ is the spatial wavenumber of the wiggler magnet and $\lambda_{w}$ the wiggler period. For an electron beam fed along the axis of the wiggler magnet, a transverse electron velocity $c \vec{\beta}_{\perp}$ is induced. It can be shown easily as

$$
\vec{\beta}_{\perp}=-\hat{y} \frac{a_{w}}{\gamma} \sin k_{w} z
$$

where $a_{w}=e B_{w 0} / m c k_{w}$ is the dimensionless wiggler strength and $\gamma$ is the relativistic factor. In the small-signal regime, the influence of the waveguide fields on the electron velocity is much smaller than the wiggler field and so the longitudinal electron velocity, $c \beta_{z}$, is related to $\beta_{\perp}$ as follows:

$$
\beta_{z}=\sqrt{1-\beta_{\perp}^{2}-\frac{1}{\gamma_{0}^{2}}}=\sqrt{1-\frac{1}{\gamma_{0}^{2}}\left(1+a_{w}^{2} \sin ^{2} k_{w} z\right)}
$$

where $\gamma_{0}$ is the initial relativistic factor of the electron beam. Alternatively

$$
\beta_{z}=\hat{\beta}_{0} \sqrt{1+\frac{a_{w r}^{2} \cos 2 k_{w} z}{\gamma_{0}^{2} \hat{\beta}_{0}^{2}}}
$$

where $\hat{\beta}_{0}=\sqrt{1-\left(1+a_{w r}^{2}\right) / \gamma_{0}^{2}}$ and $a_{w r}=a_{w} / \sqrt{2}$. As the axial electron velocity is strongly dependent on the magnitude of the wiggler field, an approximation to the first order of $a_{w r}^{2}$, as employed previously, is no longer accurate at strong wiggler field. To this end, (3) is Taylor expanded to include higher order terms

$$
\begin{aligned}
\beta_{z}=\hat{\beta}_{0}\left[1+\frac{p}{2} \cos 2 k_{w} z-\frac{p^{2}}{8} \cos ^{2} 2 k_{w} z\right. \\
\left.+\frac{p^{3}}{16} \cos ^{3} 2 k_{w} z-\frac{5 p^{4}}{128} \cos ^{4} 2 k_{w} z+\cdots\right]
\end{aligned}
$$

where $p=a_{w r}^{2} / \gamma_{0}^{2} \hat{\beta}_{0}^{2}$. To determine how many terms to keep in the above equation, the maximum possible value of $p$ needs to be estimated for second-harmonic waveguide FELs, and this in turn relates directly to the choice of the wiggler field.

It should be noted that while a large wiggler field is desirable for a strong beam-wave interaction at a given beam current it also reduces the axial electron velocity [see (3)] and, hence, undermines the maximum electron energy reduction achievable in second-harmonic waveguide FELs. Therefore, it is not always beneficial to increase the wiggler field as much as possible and, in general, the choice of the wiggler field is a compromise. Wiggler magnets used in microwave waveguide FELs typically have a period of a few centimeters and a peak on-axis field up to a few thousands Gausses [1]-[12]. For the $X$-band waveguide FEL considered previously [15], if we employ an electron beam of 95 $\mathrm{kV}$ and a strong wiggler of $B_{w 0}=0.1431 T$ and $\lambda_{w}=3.8 \mathrm{~cm}$, the $X$-band FEL system generates strong radiation between 8-12 $\mathrm{GHz}$ in a cylindrical waveguide of a diameter $2 b=3.175 \mathrm{~cm}$. In this case, it can be shown that $\hat{\beta}_{0}=0.4439$ and $p=0.4646$. If the magnetic field of the wiggler is increased further, the electron energy needs to be increased accordingly to compensate for the reduction in the effective axial electron velocity (see (2)) and to maintain strong FEL radiation over the same frequency range. Thus, $p=0.4646 \approx 0.5$ is perhaps close to what can be reasonably expected as its maximum value for microwave waveguide FELs. For second-harmonic waveguide FELs operated at higher frequencies, shorter wiggler period or/and higher electron energy are required. These requirements imply, respectively, smaller $a_{w}$ or/and larger $\gamma_{0}^{2} \hat{\beta}_{0}^{2}$ and, as such, the maximum possible value of $p$ is likely to reduce to below 0.5 . If the system parameters of the above $X$-band FEL system are considered as typical for microwave waveguide FELs, it is reasonable to assume, albeit somewhat arbitrarily, a general ceiling limit of $p=0.5$ for second-harmonic waveguide FELs operated at wavelengths from microwave to the far infrared. Substituting the 
ceiling value of $p=5$ into (4), we find that relevant coefficients are respectively $p / 2=25 \%, p^{2} / 8=3.13 \%, p^{3} / 16=0.78 \%$ and $5 p^{4} / 128=0.24 \%$. For higher-order terms (those $p^{n}$ terms with $n>4$ ), their coefficients are significantly smaller than $0.24 \%$, and so the axial electron velocity is sufficiently accurate when expressed to the fourth order of $p$ (the eighth order of the wiggler field). For our remaining analysis of second-harmonic waveguide FELs, we will use the axial electron velocity in (4) to the eighth order of the wiggler field (the fourth order of $p$ ).

With the wiggler field in the $x$ direction, electrons wiggle in the $y$ direction and so their off-axis motion subjects them to off-axis magnetic fields. Therefore, the wiggler field needs to be sufficiently uniform in the $y$ direction to ensure all electrons experiencing approximately the same magnetic field. This is also important to ensure the accuracy of (4). To this end, the width of a plannar wiggler in the $y$ direction $w$ needs to be sufficiently large. To illustrate this, we consider the plane-focusing wiggler structure used for sheet electron beams [17]. For these wiggler magnets, the $x$-directed wiggler field follows $\cosh \left(k_{y} y\right)$ variation and $k_{y}$ may be approximated by $k_{y}=2 k_{w} b / w$ with $b$ being the radius of the waveguide [17]. To keep the off-axis variation of the wiggler field within 5\% at the largest electron excursion from the axis, $y_{m}, w>6.351 k_{w} b y_{m}$ needs to be satisfied. At $p=0.5$, our numerical simulation suggests that $y_{m}<0.8 b$ and this requires $w=21 \mathrm{~cm}$. This can be much reduced by improved wiggler focusing as suggested in [18]. In addition, the requirement for (4) to be accurate is less stringent than that for the wiggler field itself as $\Delta \beta_{z} / \beta_{z}=\left(\beta_{0}^{2} / \beta_{z}^{2}-1\right)\left(\Delta \beta_{\perp} / \beta_{\perp}\right)<$ $\Delta \beta_{\perp} / \beta_{\perp}$. In the case of the $X$-band example considered above, it can be shown that $\Delta \beta_{z} / \beta_{z} \leq 0.03 \Delta \beta_{\perp} / \beta_{\perp}$ when $p \leq 0.5$. This would further lower the minimum wiggler width needed for electrons to experience sufficiently uniform wiggler field.

It is of interest to note that in (4)

$$
\begin{aligned}
\cos ^{2} 2 k_{w} z & =\frac{1}{2}\left(1+\cos 4 k_{w} z\right) \\
\cos ^{3} 2 k_{w} z & =\frac{1}{4}\left(3 \cos 2 k_{w} z+\cos 6 k_{w} z\right) \\
\cos ^{4} 2 k_{w} z & =\frac{1}{8}\left(3+4 \cos 4 k_{w} z+\cos 8 k_{w} z\right)
\end{aligned}
$$

each of which may be considered to consist of a fundamental term, $\cos 2 k_{w} z$ and its harmonic terms such as $\cos 4 k_{w} z$, $\cos 6 k_{w} z$ and $\cos 8 k_{w} z$. Thus (4) becomes

$$
\begin{aligned}
\beta_{z}=\beta_{z 0}\left(1+\chi_{0} \cos 2 k_{w} z+\chi_{1}\right. & \cos 4 k_{w} z \\
& \left.+\chi_{2} \cos 6 k_{w} z+\chi_{3} \cos 8 k_{w} z\right)
\end{aligned}
$$

where $\beta_{z 0}$ is the average of $\beta_{z}$ and $\chi_{0}>\chi_{1}>\chi_{2}>\chi_{3}$. Harmonic terms in the above equation, $\cos 2 n k_{w} z(n=2,3,4)$, represent harmonic contents in the axial electron velocity, at the fourth, sixth, and eighth harmonic of the wiggler magnet's spatial periodicity. Given that second-harmonic waveguide FELs are normally designed to couple with the $\cos 2 k_{w} z$ term of the electron velocity and that $\chi_{0}>\chi_{1}>\chi_{2}>\chi_{3}$, the contribution of harmonic terms to the beam-wave interaction is likely to be much smaller than that of the nominal $\cos 2 k_{w} z$ term. Thus, the previous equation reduces to

$$
\beta_{z}=\beta_{z 0}\left(1+\chi_{0} \cos 2 k_{w} z\right)
$$

with $\beta_{z 0}$ and $\chi_{0}$ given by

$$
\begin{aligned}
\beta_{z 0} & =\hat{\beta}_{0}\left[1-\frac{1}{16} p^{2}-\frac{15}{1024} p^{4}\right] \\
\chi_{0} & =\frac{\hat{\beta}_{0}}{\beta_{z 0}}\left[\frac{1}{2} p+\frac{3}{64} p^{3}\right] .
\end{aligned}
$$

Equation (5) is identical in format to that used previously [15], although $\chi_{0}$ and $\beta_{z 0}$ are formulated more accurately in (6) than in our previous treatment [12].

With the stable electron trajectory described in (2) and (5), the beam-wave interaction and the consequent amplification of the waveguide fields can be derived from the energy conservation equation

$$
\frac{d}{d t}\left(\gamma m c^{2}\right)=-e \vec{E}_{z} \cdot \vec{v}_{z} .
$$

where $E_{z}=E_{0} \cos \left(k_{z} z-\omega t+\varphi\right)$. If we consider only the lowest TM mode in a cylindrical waveguide, the $\mathrm{TM}_{01}$ mode, the previous equation is reduced to [15]

$$
\begin{aligned}
\frac{d\left(\gamma m c^{2}\right)}{d t}= & -e E_{0} c \beta_{z 0} \cos \left(k_{z} z-\omega t+\varphi\right) \\
& -\frac{1}{2} e E_{0} c \chi_{0} \beta_{z 0} \cos \left(2 k_{w} z+k_{z} z-\omega t+\varphi\right) \\
& -\frac{1}{2} e E_{0} c \chi_{0} \beta_{z 0} \cos \left(2 k_{w} z-k_{z} z-\omega t+\varphi\right) .
\end{aligned}
$$

Similar to the usual treatment of waveguide free electron lasers, the third term may be ignored in comparison with the second term. On the other hand, the first term is the new nonresonant term and it needs to be included [15]. Thus, (7) becomes

$$
\begin{aligned}
\frac{d \gamma}{d z}=-\frac{\chi_{0}}{2} \frac{\omega a_{s}}{c} \frac{\beta_{z 0}}{\beta_{z}} \cos ( & \left.2 k_{w} z+k_{z} z-\omega t+\varphi\right) \\
& -\frac{\omega a_{s}}{c} \frac{\beta_{z 0}}{\beta_{z}} \cos \left(k_{z} z-\omega t+\varphi\right)
\end{aligned}
$$

where $a_{s}=e E_{0} / \mathrm{mcw}$. It should be noted that $t$ on the right-hand side of the above equation is related to $z$ via the axial electron velocity and the latter is usually a function of time. Thus, an exact integral of the above equation is in general mathematically complicated and does not necessarily lead to an analytically convenient formula. To enable a simplified analytical integration, we consider the usual technique of approximating the $z-t$ relation by means of the average axial electron velocity. For most waveguide FELs, this simplification is usually realized through the following approximation

$$
t=\int_{0}^{L} \frac{d z}{c \beta_{z}} \approx \frac{z}{c \beta_{z 0}}
$$

where $\beta_{z 0}$ is the average axial electron velocity given in (6a) and $L$ the wiggler length. However, as the wiggler field is strong, the previous approximation may not be sufficiently accurate. To assess this, the spatial integral in the previous equation is calculated from Taylor expanding the reciprocal of (3) to the fourth order of $p$ to give

$$
\frac{1}{L} \int_{0}^{L} \frac{d z}{\beta_{z}} \equiv\left\langle\frac{1}{\beta_{z}}\right\rangle=\frac{1}{\hat{\beta}_{0}}\left[1+\frac{3}{16} p^{2}+\frac{105}{1024} p^{4}\right]
$$


where $\langle\cdots\rangle$ represents a spatial average. Comparison of the above equation with $1 / \beta_{z 0}$ suggests that the average of $1 / \beta_{z}$ is not analytically the same as $1 / \beta_{z 0}$ at large wiggler field. As an illustration, it can be shown that $\left\langle 1 / \beta_{z}\right\rangle$ is 2.3545 and $1 / \beta_{z 0}$ is 2.2849 at $p=0.5$. Although their difference is only about $3 \%$, the FEL resonant condition is likely to be considerably altered if the axial electron velocity is shifted by $3 \%$. For this reason, (9) is employed throughout our gain formulation.

Substituting (9) into (8) gives

$$
\frac{d \gamma}{d z}=-\frac{\chi_{0}}{2} \frac{\omega a_{s}}{c}\left\langle\frac{\beta_{z 0}}{\beta_{z}}\right\rangle \cos \Delta k z-\frac{\omega a_{s}}{c}\left\langle\frac{\beta_{z 0}}{\beta_{z}}\right\rangle \cos \Delta \tilde{k} z
$$

where $\Delta k$ and $\Delta \tilde{k}$ are respectively the resonant and nonresonant FEL detuning parameters given by [15]

$$
\begin{aligned}
& \Delta k=2 k_{w}+k_{z}-\frac{\omega}{c}\left\langle\frac{1}{\beta_{z}}\right\rangle \\
& \Delta \tilde{k}=k_{z}-\frac{\omega}{c}\left\langle\frac{1}{\beta_{z}}\right\rangle .
\end{aligned}
$$

Integrating (10) directly over $L$ yields the energy change of a single electron

$$
\begin{aligned}
\Delta \gamma_{1}=-\frac{\omega a_{s} \chi_{0} L}{2 c} & \left\langle\frac{\beta_{z 0}}{\beta_{z}}\right\rangle \frac{\sin \left(\frac{\Delta k L}{2}\right)}{\frac{\Delta k L}{2}} \cos \left(\frac{\Delta k L}{2}+\varphi\right) \\
- & \frac{\omega a_{s} L}{c}\left\langle\frac{\beta_{z 0}}{\beta_{z}}\right\rangle \frac{\sin \left(\frac{\Delta \tilde{k} L}{2}\right)}{\frac{\Delta \tilde{k} L}{2}} \cos \left(\frac{\Delta \tilde{k} L}{2}+\varphi\right)
\end{aligned}
$$

to the first order of the waveguide field. Using Madey's theorem [19]

$$
\left\langle\Delta \gamma_{2}\right\rangle_{p}=\frac{1}{2} \frac{d}{d \gamma}\left\langle\left(\Delta \gamma_{1}\right)^{2}\right\rangle_{p}
$$

with $\langle\cdots\rangle_{p}$ an average over the initial electron phase, the net energy change of the electron beam is obtained, to the second order of the waveguide field, as follows

$$
\begin{aligned}
\left\langle\Delta \gamma_{2}\right\rangle_{p}= & \frac{\chi_{0}}{4}\left[\frac{\omega a_{s} L}{c}\right]^{2}\left\langle\frac{\beta_{z 0}}{\beta_{z}}\right\rangle^{2} \\
& \cdot\left[\operatorname{sinc} x \frac{d \tilde{x}}{d \gamma} \frac{d \operatorname{sinc} \tilde{x}}{d \tilde{x}}+\operatorname{sinc} \tilde{x} \frac{d x}{d \gamma} \frac{d \operatorname{sinc} x}{d x}\right] \\
& +\frac{1}{4}\left[\frac{\omega a_{s} L}{c}\right]^{2}\left\langle\frac{\beta_{z 0}}{\beta_{z}}\right\rangle^{2} \\
& \cdot\left[\frac{d \tilde{x}}{d \gamma} \frac{d \operatorname{sinc}^{2} \tilde{x}}{d \tilde{x}}+\frac{\chi_{0}^{2}}{4} \frac{d x}{d \gamma} \frac{d \operatorname{sinc}^{2} x}{d x}\right]
\end{aligned}
$$

where $x=\Delta k L / 2$ and $\tilde{x}=\Delta \tilde{k} L / 2$. Let $P_{g}$ the propagating power of the $\mathrm{TM}_{01}$ mode, the power gain is then given by

$$
G=\frac{\left\langle\Delta \gamma_{2}\right\rangle_{p} m c^{2} \frac{I}{|e|}}{P_{g}} .
$$

Note that

$$
\frac{d x}{d \gamma}=\frac{d \tilde{x}}{d \gamma}=\frac{\omega L}{2 c}\left\langle\frac{1}{\beta_{z}}\right\rangle^{3} \frac{1+a_{w r}^{2}}{\gamma_{0}^{3}}
$$
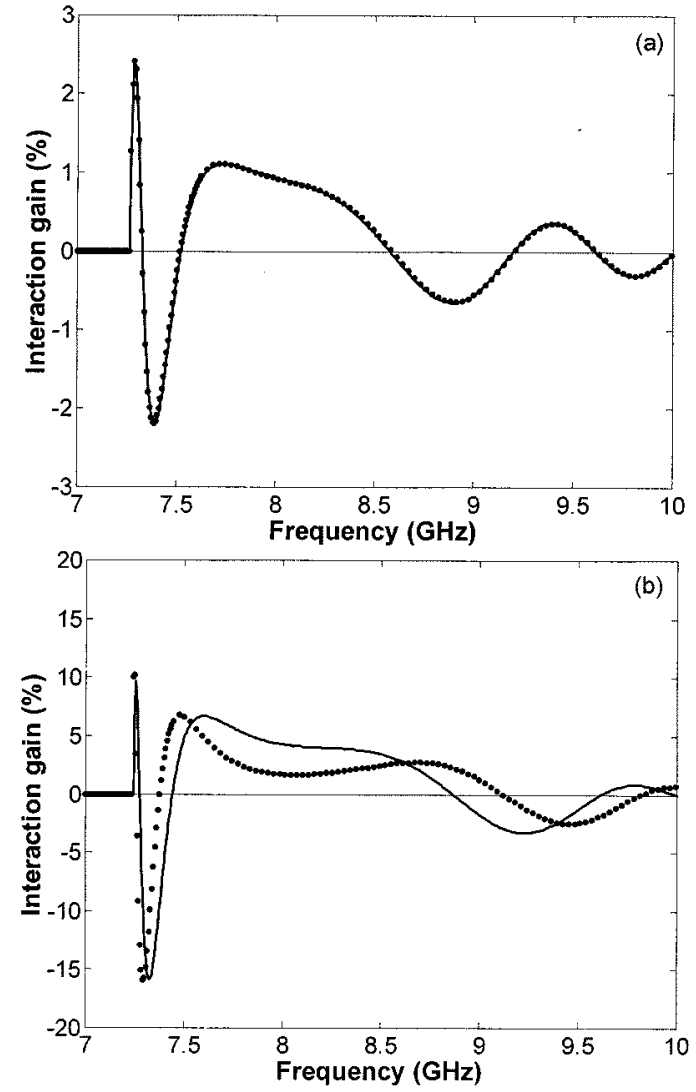

Fig. 1. Small-signal gain as a function of frequency calculated from (14) (solid line) and [15] (dots) for (a) $\chi_{0}=0.02(p=0.04)$ and (b) $\chi_{0}=0.1(p=0.22)$.

the interaction gain of (13) is derived from (12) as

$$
\begin{aligned}
G=G_{0} \frac{k_{c}^{2} L}{k_{z}}\{ & {\left[\frac{d \operatorname{sinc}^{2} \tilde{x}}{d \tilde{x}}+\frac{\chi_{0}^{2}}{4} \cdot \frac{d \operatorname{sinc}^{2} x}{d x}\right] } \\
& \left.+\chi_{0}\left[\operatorname{sinc} x \cdot \frac{d \operatorname{sinc} \tilde{x}}{d \tilde{x}}+\operatorname{sinc} \tilde{x} \cdot \frac{d \operatorname{sinc} x}{d x}\right]\right\}
\end{aligned}
$$

where the cutoff wavenumber of the $\mathrm{TM}_{01}$ mode $k_{c}=2.4048 / b$ and $G_{0}$ is given by

$$
G_{0}=-\frac{1}{8 J_{1}^{\prime 2}(2.4048)}\left[Z_{0} \frac{I e}{m c^{2}}\right] \frac{L^{2} \beta_{z 0}^{2}}{A_{e m}}\left\langle\frac{1}{\beta_{z}}\right\rangle^{5} \frac{1+a_{w r}^{2}}{\gamma_{0}^{3}} .
$$

with $A_{e m}=\pi b^{2} / 2$ being the effective cross-sectional area of the $\mathrm{TM}_{01}$ mode. The interaction gain formulated in (14) and (15) is very similar to that reported previously [15] in terms of format. Their main difference lies in the formulation of $\chi_{0}$, $\beta_{z 0}$ and $\left\langle 1 / \beta_{z}\right\rangle$. At the limit of $p \ll 1$, the small wiggler field approximation becomes valid and the interaction gain in (14) can be shown to reduce to that formulated previously [15]. To illustrate quantitatively their difference at different wiggler fields, (14) and the gain formula in [15] are used to calculate the small-signal gain of an $X$-band system of $\lambda_{w}=3.8 \mathrm{~cm}$, $L=11 \lambda_{w}$ and $I=20 \mathrm{~mA}$ but for two different values of $\chi_{0}, \chi_{0}=0.02(p=0.04)$ and $\chi_{0}=0.1(p=0.22)$. As shown in Fig. 1, the two gain formulas produce almost the same gain curve when the wiggler field is week at $\chi_{0}=0.02$ and the electron voltage is $V_{e}=53.2 \mathrm{kV}$. However, when the wiggler field is increased to $\chi_{0}=0.1$ with the electron voltage adjusted 
to $V_{e}=68 \mathrm{kV}$, there is a significant difference and the previously formulated gain is no longer applicable. Equation (14) is, therefore, applicable to a much wider range of the wiggler field. Also, the above comparison suggests that while the interaction gain is increased by a factor of five in Fig. 1(b) the electron energy required is also increased by $(68 / 53.2-1)=28 \%$. Thus, when second-harmonic waveguide FELs are designed to operate at strong wiggler field for a large interaction gain, care must be exercised not to incur too large an increase in electron energy.

It is worth noting that under the free space condition (when $b \rightarrow \infty$ ) the interaction gain of (14) becomes zero through its dependence upon $k_{c}^{2} / A_{e m} \propto 1 / b^{4}$. This is consistent with the fact that the electromagnetic wave becomes a TEM mode in free space and so it does not have a longitudinal electric field component to support its interaction with the longitudinal electron velocity.

It is also of interest to consider the interaction gain of (14) for the wiggler-free case. By setting $a_{w}$ to zero (thus $\chi_{0}=0$ ) and $\lambda_{w}$ to infinity in (14), the interaction gain does not always reduce to zero, although it is very small because of the very large $\tilde{x}$ when $\lambda_{w}=\infty$. This can be understood from the fact that there are, in general, a finite energy exchange between an electron and a travelling wave even when they are not in resonance in the absence of a wiggler magnet. If they are not in resonance, the electromagnetic wave bypasses the electron in the electron-rest frame and the electron experiences acceleration in one half-cycle of the wave and deceleration in the next half cycle. When the electromagnetic wave bypasses the electron by a complete number of full cycles, the energy gained is cancelled out completely by the energy lost and the net energy exchange is zero. This corresponds to the case when $\tilde{x}=0$. However, if the wave bypasses the electron by incomplete cycles, finite energy exchange is expected between electrons and the electromagnetic wave.

\section{Gain Formulation Using Perturbation TheOry}

Madey's theorem simplifies the mathematical processes to obtain the interaction gain of conventional free electron laser systems that rely on a strong coupling between the transverse electron velocity and the transverse electric field component of the radiation field [19]. As shown in (11), Madey's theorem relates the net electron energy change at the second order of the radiation field to the electron energy change at the first order of the radiation field, thus eliminating the need of direct formulation of the former. This is clearly mathematically efficient. However as Madey's theorem has yet to be established for second-harmonic waveguide FELs, our gain formulation in the preceding section is effectively based on an hypothesis. An independent gain formulation is, therefore, important given that the significant difference in interaction behaviors between second-harmonic waveguide FELs and their conventional counterparts may compromise the applicability of Madey's theorem to these new waveguide FELs. To calculate their interaction gain without recourse to Madey's theorem, their electron energy change needs to be formulated directly to the second order of the waveguide field [20].
Under the combined field of the wiggler magnet and the $\mathrm{TM}_{01}$ mode, the electron trajectory is described by its equation of motion

$$
\frac{d}{d t}\left(\gamma m \vec{v}_{z}\right)=-e\left[\vec{E}_{z}+\vec{v}_{y} \times \vec{B}_{w}\right] .
$$

In the 1-D limit and with (1) and (2), the above equation reduces to

$$
\begin{aligned}
\frac{d}{d t}\left(\gamma \vec{\beta}_{z}\right)=-\hat{z}\left[\omega a_{s} \cos \left(k_{z} z-\omega t+\varphi\right)\right. & \\
& \left.+\frac{a_{w r}^{2} c k_{w}}{\gamma} \sin 2 k_{w} z\right] .
\end{aligned}
$$

Electron energy change is described by (8), which may be rewritten as

$$
\frac{d \gamma}{d t}=-\omega a_{s} \beta_{z 0}\left[\frac{\chi_{0}}{2} \cos \Phi+\cos \tilde{\Phi}\right]
$$

where $\tilde{\Phi}=k_{z} z-\omega t+\varphi$ is the phase of the $\mathrm{TM}_{01}$ mode and $\Phi=\left(2 k_{w}+k_{z}\right) z-\omega t+\varphi$ is the phase of the ponderomotive potential associated with the desired coupling between $E_{z}$ of the $\mathrm{TM}_{01}$ mode and the $\cos k_{w} z$ component of the axial electron velocity in second-harmonic waveguide FELs. It is shown in (17) that the electron energy change consists of two terms. The first term on the right-hand side represents the preferred beam-wave interaction at the second harmonic of the wiggler's periodicity. System parameters are usually chosen such that $\Phi$ remains relatively unchanged during the electron passage through the wiggler magnet. As a result, electrons are kept decelerated in the ponderomotive potential and this first term in (17) makes an accumulatively significant and dominating contribution to the net electron energy change. On the other hand, the second term on the right-hand side of (17) represents the electron interaction with the waveguide field in the absence of the wiggler magnet. Since it is impossible to synchronize the electron beam and the waveguide field without the wiggler in a smoothbore waveguide, $\tilde{\Phi}$ varies quickly and so the $\cos \tilde{\Phi}$ term oscillates rapidly between its positive and negative bounds as the electron beam transverses through the wiggler magnet. As a result, electrons gain energy from and loss energy to the waveguide fields alternatively and the total net energy exchange through this second term is likely to be very small. As highlighted in [12] however, it is important to include this second term in our gain formulation.

To describe the beam-wave interaction in the ponderomotive potential, we employ the technique used in [20] and consider the second time derivative of $\Phi$

$$
\ddot{\Phi} \equiv \frac{d^{2} \Phi}{d t^{2}}=\left(2 k_{w}+k_{z}\right) c \frac{d \beta_{z}}{d t} .
$$

$d \beta_{z} / d t$ is obtained from substituting (17) into (16)

$$
\begin{aligned}
\frac{d \beta_{z}}{d t}=- & \frac{1-\beta_{z}^{2}}{\gamma \beta_{z}} \beta_{z 0} \omega a_{s} \frac{\chi_{0}}{2} \cos \Phi \\
& \quad-\frac{1-\beta_{z}^{2}}{\gamma \beta_{z}} \beta_{z 0} \omega a_{s} \cos \tilde{\Phi}-\frac{a_{w r}^{2} c k_{w}}{\gamma^{2}} \sin 2 k_{w} z
\end{aligned}
$$

and so

$$
\ddot{\Phi}=-\Omega^{2} \cos \Phi-\tilde{\Omega}^{2} \cos \tilde{\Phi}-m \sin 2 k_{w} z
$$


with

$$
\begin{aligned}
\Omega^{2} & =\left(2 k_{w}+k_{z}\right) \omega a_{s} c \chi_{0} \frac{1-\beta_{z}^{2}}{2 \gamma \beta_{z}} \beta_{z 0} \\
& \approx\left(2 k_{w}+k_{z}\right) \omega a_{s} c \chi_{0} \frac{1+a_{w r}^{2}}{2 \gamma_{0}^{3}}\left\langle\frac{1}{\beta_{z}}\right\rangle \beta_{z 0} \\
\tilde{\Omega}^{2} & =\left(2 k_{w}+k_{z}\right) c \frac{1-\beta_{z}^{2}}{\gamma \beta_{z}} \beta_{z 0} \omega a_{s}=\frac{2}{\chi_{0}} \Omega^{2} \\
m & =\frac{a_{w r}^{2} c^{2} k_{w}\left(2 k_{w}+k_{z}\right)}{\gamma^{2}} .
\end{aligned}
$$

Equation (19) is the pendulum equation of electrons in the ponderomotive potential. It is of interest to note the second and third terms on the right-hand side of (19) which describe the individual influence of the waveguide field and the wiggler magnet, respectively. Since these two terms are absent in the pendulum equation for conventional FELs [20], the solution of (19) is likely to be more complex and the electron energy exchange with the radiation field is likely to contain significantly new features in second-harmonic waveguide FELs.

Comparing (17) with (19), we find that

$$
\frac{d^{2} \Phi}{d t^{2}}=\frac{\left(2 k_{w}+k_{z}\right) c\left(1-\beta_{z}^{2}\right)}{\gamma \beta_{z}} \frac{d \gamma}{d t}-m \sin 2 k_{w} z
$$

or alternatively in a more useful format of

$$
\frac{d \gamma}{d t}=\frac{\omega a_{s} \beta_{z 0} \chi_{0}}{2 \Omega^{2}} \ddot{\Phi}+\frac{a_{w r}^{2} k_{w} c}{\gamma} \frac{\beta_{z}}{1-\beta_{z}^{2}} \sin 2 k_{w} z
$$

To integrate the above equation, we assume that $\beta_{z} / \gamma\left(1-\beta_{z}^{2}\right)$ is approximately constant and at the exit of the wiggler this approximation gives

$$
\gamma-\gamma_{0}=\frac{\omega a_{s} \beta_{z 0} \chi_{0}}{2 \Omega^{2}}[\dot{\Phi}-\dot{\Phi}(0)]
$$

where the second term in (22) has been integrated to zero and $\dot{\Phi} \equiv d \Phi / d t$. As

$$
\dot{\Phi}(0)=\left(2 k_{w}+k_{z}\right) v_{z}(0)-\omega \equiv \Delta \omega
$$

(23) becomes

$$
\Delta \gamma=\omega a_{s} \beta_{z 0} \chi_{0} \frac{[\dot{\Phi}-\Delta \omega]}{2 \Omega^{2}} .
$$

Thus, $\Delta \gamma$ and, hence, the interaction gain can be formulated when $\dot{\Phi}(t)$ is known. The formulation of $\dot{\Phi}(t)$ may be obtained from (19), which may be rewritten as

$$
\begin{aligned}
\ddot{\Phi}=-\Omega^{2} \cos \Phi-\tilde{\Omega}^{2} \cos 2 k_{w} z \cos \Phi \\
-\tilde{\Omega}^{2} \sin \Phi \sin 2 k_{w} z-m \sin 2 k_{w} z .
\end{aligned}
$$

In general, the solution of (26) for $\ddot{\Phi}(t)$ requires elliptical integrals and the relevant mathematical formulation is rather complex. This is particularly so when the radiation field is large and its influence on the $z-t$ relationship has to be included in the expression of $\Phi$ and $\tilde{\Phi}$ in (26). In the small-signal regime however, the phase of the ponderomotive potential may be considered to be determined predominately by the stable electron trajectory of (2) and (5) with the influence of the radiation field treated as small perturbations. Therefore, $\Phi$ may be Taylor expanded to different orders of the radiation field, or more conveniently that of $\Omega^{2}$ as follows:

$$
\Phi=\Phi_{0}+\Phi_{1}+\Phi_{2}+\ldots
$$

where $\Phi_{n} \propto\left(\Omega^{2}\right)^{n}$ and $\Phi_{n} \propto\left(\tilde{\Omega}^{2}\right)^{n}$ with $n=1,2,3, \ldots$ Thus, $\ddot{\Phi}$ can be expressed as

$$
\ddot{\Phi}=\ddot{\Phi}_{0}+\ddot{\Phi}_{1}+\ddot{\Phi}_{2}+\ldots
$$

Equation (26) can be shown to deduce

$$
\begin{aligned}
\ddot{\Phi} & \approx\left[-\tilde{\Omega}^{2} \cos 2 k_{w} z-\Omega^{2}\right]\left(\cos \Phi_{0}-\sin \Phi_{0} \sin \Phi_{1}\right) \\
& -\tilde{\Omega}^{2}\left(\sin \Phi_{0}+\cos \Phi_{0} \sin \Phi_{1}\right) \sin 2 k_{w} z-m \sin 2 k_{w} z .
\end{aligned}
$$

Substituting (27) into the left-hand side of (28) gives

$$
\begin{aligned}
\ddot{\Phi}_{0}= & -m \sin 2 k_{w} z \\
\ddot{\Phi}_{1}= & -\Omega^{2} \cos \Phi_{0}-\tilde{\Omega}^{2} \cos 2 k_{w} z \cos \Phi_{0} \\
& -\tilde{\Omega}^{2} \sin 2 k_{w} z \sin \Phi_{0} \\
\ddot{\Phi}_{2}= & \Omega^{2} \sin \Phi_{0} \sin \Phi_{1}+\tilde{\Omega}^{2} \cos 2 k_{w} z \sin \Phi_{0} \sin \Phi_{1} \\
& -\tilde{\Omega}^{2} \sin 2 k_{w} z \cos \Phi_{0} \sin \Phi_{1} .
\end{aligned}
$$

So the solution of (26) can be obtained by solving the previous three equations. Since

$$
\frac{d^{2} \Phi_{0}}{d t^{2}}=\frac{d z}{d t} \frac{d}{d z}\left[\frac{d \Phi_{0}}{d t}\right]=c \beta_{z} \frac{d}{d z}\left[\frac{d \Phi_{0}}{d t}\right]
$$

the first equation of (30) becomes

$$
\frac{d}{d z}\left[\frac{d \Phi_{0}}{d t}\right]=-\frac{a_{w r}^{2} k_{w} c\left(2 k_{w}+k_{z}\right)}{\gamma^{2}}\left\langle\frac{1}{\beta_{z}}\right\rangle \sin 2 k_{w} z
$$

where $\left(1 / \beta_{z}\right)$ has been replaced by $\left\langle 1 / \beta_{z}\right\rangle$, as an approximation, to permit a direct integral of (32). With the initial condition specified in (24), we have

$$
\frac{d \Phi_{0}}{d t}=-\frac{a_{w r}^{2} c\left(2 k_{w}+k_{z}\right)}{2 \gamma^{2}}\left\langle\frac{1}{\beta_{z}}\right\rangle\left(1-\cos 2 k_{w} z\right)+\Delta \omega .
$$

Note that from the above equation $\dot{\Phi}_{0}=\Delta \omega$ at $z=L$. Again, we use $\left\langle 1 / \beta_{z}\right\rangle$ to approximate $\left(1 / \beta_{z}\right)$ to obtain the integral of the above equation with respect to $z$ as

$$
\begin{aligned}
& \Phi_{0}(z)-\Phi_{0}(0)=\frac{\Delta \omega z}{c}\left\langle\frac{1}{\beta_{z}}\right\rangle- \frac{a_{w r}^{2}\left(2 k_{w}+k_{z}\right)}{4 \gamma^{2} k_{w}}\left\langle\frac{1}{\beta_{z}}\right\rangle^{2} \\
& \cdot\left(2 k_{w} z-\sin 2 k_{w} z\right)
\end{aligned}
$$

From (24), the first two terms on the right-hand side of (32) become

$$
\begin{aligned}
& \frac{\Delta \omega z}{c}\left\langle\frac{1}{\beta_{z}}\right\rangle-\frac{a_{w r}^{2}\left(2 k_{w}+k_{z}\right)}{4 \gamma^{2} k_{w}}\left\langle\frac{1}{\beta_{z}}\right\rangle^{2} 2 k_{w} z \\
& =\left\langle\frac{1}{\beta_{z}}\right\rangle z\left[\left(2 k_{w}+k_{z}\right) \beta_{z}(0)-\frac{\omega}{c}-\left(2 k_{w}+k_{z}\right) \frac{p}{2}\left\langle\frac{\hat{\beta}_{0}^{2}}{\beta_{z}}\right\rangle\right]
\end{aligned}
$$


where $p=a_{w r}^{2} / \gamma_{0}^{2} \hat{\beta}_{0}^{2}$ has been used. From (5), (6), and (9), we have

$$
\begin{aligned}
&\left(2 k_{w}+k_{z}\right) \beta_{z}(0)\left\langle\frac{1}{\beta_{z}}\right\rangle-\left(2 k_{w}+k_{z}\right) \frac{p}{2}\left\langle\frac{\hat{\beta}_{0}^{2}}{\beta_{z}}\right\rangle\left\langle\frac{1}{\beta_{z}}\right\rangle \\
&=\left(2 k_{w}+k_{z}\right)\left\langle\frac{1}{\beta_{z}}\right\rangle \\
& \\
& \cdot\left[\beta_{z 0}\left(1+\frac{\hat{\beta}_{0}}{\beta_{z 0}} \frac{p}{2}\right)-\frac{p}{2}\left[1+\frac{3 p^{2}}{16}\right] \hat{\beta}_{0}\right] \\
&=\left(2 k_{w}+k_{z}\right)\left\langle\frac{1}{\beta_{z}}\right\rangle \\
& \\
& \cdot\left[\hat{\beta}_{0}\left(1-\frac{p^{2}}{16}\right)-\hat{\beta}_{0} \frac{p}{2}-\frac{p}{2} \hat{\beta}_{0}-\frac{3 p^{3}}{32} \hat{\beta}_{0}\right] \\
&=\left(2 k_{w}+k_{z}\right) \frac{1}{\hat{\beta}_{0}}\left[1+\frac{3 p^{2}}{16}\right]\left[\hat{\beta}_{0}\left(1-\frac{p^{2}}{16}-\frac{3 p^{3}}{32}\right)\right] \\
& \approx\left(2 k_{w}+k_{z}\right) .
\end{aligned}
$$

Hence, in (32)

$\frac{\Delta \omega z}{c}\left\langle\frac{1}{\beta_{z}}\right\rangle-\frac{a_{w r}^{2}\left(2 k_{w}+k_{z}\right)}{4 \gamma^{2} k_{w}}\left\langle\frac{1}{\beta_{z}}\right\rangle^{2} 2 k_{w} z=\left(2 k_{w}+k_{z}\right) z$

and (32) becomes

$$
\begin{aligned}
\Phi_{0}(z)-\Phi_{0}(0)= & {\left[\left(2 k_{w}+k_{z}\right)-\frac{\omega}{c}\left\langle\frac{1}{\beta_{z}}\right\rangle\right] z } \\
& -\frac{p}{2}\left\langle\frac{\hat{\beta}_{0}}{\beta_{z}}\right\rangle^{2} \sin 2 k_{w} z \\
= & \Delta k z-\frac{p}{2}\left\langle\frac{\hat{\beta}_{0}}{\beta_{z}}\right\rangle^{2} \sin 2 k_{w} z .
\end{aligned}
$$

The first term in the previous equation describes the desired phase evolution of the ponderomotive potential while the second term represents a small correction. The latter describes detailed features of electron trajectory that are not included in the simplified $z-t$ relationship of $t=z\left\langle 1 / \beta_{z}\right\rangle / c$. As this second term oscillates between its positive and negative bounds, its accumulated effects are likely to very small indeed. Mathematically on the other hand, its inclusion in (32) makes it analytically difficult to progress our gain formulation. From the above two considerations, we ignore this second term as an approximation. Note that $\Phi_{0}(0)=\varphi$, the above equation becomes

$$
\Phi_{0}(z)=\Delta k z+\varphi .
$$

The second equation in (29) may be rewritten as

$$
\ddot{\Phi}_{1}=-\tilde{\Omega}^{2} \cos \left(\Phi_{0}-2 k_{w} z\right)-\Omega^{2} \cos \Phi_{0} .
$$

Substituting (33) into (34) and, subsequently, integrating the latter gives

$$
\begin{aligned}
\frac{d \Phi_{1}}{d t}=-\frac{\tilde{\Omega}^{2}}{c \Delta \tilde{k}} & \left\langle\frac{1}{\beta_{z}}\right\rangle[\sin (\Delta \tilde{k} z+\varphi)-\sin \varphi] \\
& -\frac{\Omega^{2}}{c \Delta k}\left\langle\frac{1}{\beta_{z}}\right\rangle[\sin (\Delta k z+\varphi)-\sin \varphi]
\end{aligned}
$$

where the initial condition of $\dot{\Phi}_{1}(0)=0$ and the relationship of $\Delta \tilde{k}=\Delta k-2 k_{w}$ have been used. It should be mentioned also that $\left(1 / \beta_{z}\right)$ is again replaced by $\left\langle 1 / \beta_{z}\right\rangle$ in the integral from (34) to (35), similar to the technique used in arriving at (31). By using its initial condition of $\Phi_{1}(0)=0, \Phi_{1}(z)$ is obtained from (35) as

$$
\begin{aligned}
\Phi_{1}(z)= & -\left[\frac{\tilde{\Omega}}{c \Delta \tilde{k}}\right]^{2}\left\langle\frac{1}{\beta_{z}}\right\rangle^{2} \\
& \cdot[\cos \varphi-\cos (\Delta \tilde{k} z+\varphi)-\Delta \tilde{k} z \sin \varphi] \\
& -\left[\frac{\Omega}{c \Delta k}\right]^{2}\left\langle\frac{1}{\beta_{z}}\right\rangle^{2} \\
& \cdot[\cos \varphi-\cos (\Delta k z+\varphi)-\Delta k z \sin \varphi] .
\end{aligned}
$$

Similar to (34), the third equation of (29) may be rewritten as

$$
\ddot{\Phi}_{2} \approx \tilde{\Omega}^{2} \Phi_{1} \sin (\Delta \tilde{k} z+\varphi)+\Omega^{2} \Phi_{1} \sin (\Delta k z+\varphi)
$$

where $\sin \Phi_{1} \approx \Phi_{1}$ has been approximated. With (36), (37) becomes

$$
\begin{array}{r}
\frac{d \Phi_{2}}{d t}=\frac{\tilde{\Omega}^{2}}{c}\left\langle\frac{1}{\beta_{z}}\right\rangle \int_{0}^{z} \Phi_{1} \sin (\Delta \tilde{k} z+\varphi) d z+\frac{\Omega^{2}}{c}\left\langle\frac{1}{\beta_{z}}\right\rangle \\
\cdot \int_{0}^{z} \Phi_{1} \sin (\Delta k z+\varphi) d z
\end{array}
$$

Further formulation of the previous equation involves the use of (36) and in principle this leads to the formulation of the energy change of a single electron to the second order of the waveguide fields. The overall energy change of the electron beam can then be obtained from an average over the electrons' initial phase. Mathematically, however, it is more efficient to perform the electron phase average to (38) directly before the spatial integrals in (38) are formulated. To relate this to the net energy change of the electron beam, we note that from (25)

$$
\begin{aligned}
& \frac{1}{2 \pi} \int_{0}^{2 \pi} \Delta \gamma d \varphi=\frac{\omega a_{s} \beta_{z 0} \chi_{0}}{2 \Omega^{2}} \\
& \cdot\left[\left\langle\dot{\Phi}_{0}\right\rangle_{p}+\left\langle\dot{\Phi}_{1}\right\rangle_{p}+\left\langle\dot{\Phi}_{2}\right\rangle_{p}+\cdots-\Delta \omega\right]
\end{aligned}
$$

where $\langle\cdots\rangle_{p}$ represents an average over the initial electron phase and

$$
\begin{aligned}
\left\langle\dot{\Phi}_{0}\right\rangle_{p}= & \frac{1}{2 \pi} \int_{0}^{2 \pi} \Delta \omega d \varphi=\Delta \omega \\
\left\langle\dot{\Phi}_{1}\right\rangle_{p}= & -\frac{\tilde{\Omega}^{2}}{2 \pi c \Delta \tilde{k}}\left\langle\frac{1}{\beta_{z}}\right\rangle \\
& \cdot \int_{0}^{2 \pi}[\sin (\Delta \tilde{k} z+\varphi)-\sin \varphi] d \varphi \\
& -\frac{\Omega^{2}}{2 \pi c \Delta k}\left\langle\frac{1}{\beta_{z}}\right\rangle \\
& \cdot \int_{0}^{2 \pi}[\sin (\Delta k z+\varphi)-\sin \varphi] d \varphi=0 .
\end{aligned}
$$


Letting $\tilde{\Lambda}=\tilde{\Omega}\left\langle 1 / \beta_{z}\right\rangle / c \Delta \tilde{k}$ and $\Lambda=\Omega\left\langle 1 / \beta_{z}\right\rangle / c \Delta k$, we have from (38) (The equation at the bottom of the page.) after (36) has been used and subsequently phase integral performed. With the spatial integrals from $z=0$ to $z=L$, the previous equation reduces to

$$
\begin{aligned}
\left\langle\dot{\Phi}_{2}(L)\right\rangle_{p}= & \Omega^{4}\left(\frac{L}{2 c}\right)^{3}\left\langle\frac{1}{\beta_{z}}\right\rangle^{3}\left[\frac{d}{d x} \frac{\sin ^{2} x}{x^{2}}+\frac{4}{\chi_{0}^{2}} \frac{d}{d \tilde{x}} \frac{\sin ^{2} \tilde{x}}{\tilde{x}^{2}}\right] \\
& +\frac{2 \Omega^{4}}{\chi_{0}}\left(\frac{L}{2 c}\right)^{3}\left\langle\frac{1}{\beta_{z}}\right\rangle^{3} \\
& . \frac{\sin \tilde{x}}{x}\left[\frac{d}{d \tilde{x}} \frac{\sin \tilde{x}}{\tilde{x}}+\frac{\cos \tilde{x}}{\tilde{x}}-\frac{\sin \tilde{x}}{x \tilde{x}}\right] \\
& +\frac{2 \Omega^{4}}{\chi_{0}}\left(\frac{L}{2 c}\right)^{3}\left\langle\frac{1}{\beta_{z}}\right\rangle^{3} \\
& . \frac{\sin x}{\tilde{x}}\left[\frac{d}{d x} \frac{\sin x}{x}+\frac{\cos x}{x}-\frac{\sin x}{x \tilde{x}}\right] .
\end{aligned}
$$

Hence, from (39)

$$
\begin{aligned}
\left\langle\Delta \gamma_{2}\right\rangle_{p}= & \frac{\chi_{0} \Omega^{2} \omega a_{s}}{2}\left(\frac{L}{2 c}\right)^{3}\left\langle\frac{1}{\beta_{z}}\right\rangle^{3} \\
& \cdot\left[\frac{d}{d x} \frac{\sin ^{2} x}{x^{2}}+\frac{4}{\chi_{0}^{2}} \frac{d}{d \tilde{x}} \frac{\sin ^{2} \tilde{x}}{\tilde{x}^{2}}+\frac{2}{\chi_{0}} f(x, \tilde{x})\right]
\end{aligned}
$$

where

$$
\begin{aligned}
f(x, \tilde{x})=\frac{\sin \tilde{x}}{x}\left[\frac{d}{d \tilde{x}} \frac{\sin \tilde{x}}{\tilde{x}}+\frac{\cos \tilde{x}}{\tilde{x}}-\frac{\sin \tilde{x}}{x \tilde{x}}\right] \\
+\frac{\sin x}{\tilde{x}}\left[\frac{d}{d x} \frac{\sin x}{x}+\frac{\cos x}{x}-\frac{\sin x}{x \tilde{x}}\right] .
\end{aligned}
$$

Consequently

$$
\begin{aligned}
\left\langle\Delta \gamma_{2}\right\rangle_{p}= & \frac{1}{8}\left(\frac{\omega a_{s} L}{c}\right)^{2}\left\langle\frac{1}{\beta_{z}}\right\rangle^{4} \frac{1+a_{w r}^{2}}{\gamma_{0}^{3}}\left(2 k_{w}+k_{z}\right) L \beta_{z 0}^{2} \\
& \times\left[\frac{d}{d \tilde{x}} \frac{\sin ^{2} \tilde{x}}{\tilde{x}^{2}}+\frac{\chi_{0}^{2}}{4} \frac{d}{d x} \frac{\sin ^{2} x}{x^{2}}+\frac{\chi_{0}}{2} f(x, \tilde{x})\right] .
\end{aligned}
$$

By substituting (42) into (13), we obtain the following smallsignal gain:

$$
\begin{aligned}
G_{p}= & -\frac{1}{8 J_{1}^{\prime 2}(2.4048)}\left[Z_{0} \frac{I e}{m c^{2}}\right] \frac{L^{2}}{A_{e m}} \frac{c\left(2 k_{w}+k_{z}\right)}{\omega} \\
& \times\left\langle\frac{1}{\beta_{z}}\right\rangle^{4} \frac{1+a_{w r}^{2}}{\gamma_{0}^{3}} \frac{k_{c}^{2} L}{k_{z}} \beta_{z 0}^{2} \\
& \times\left[\frac{d}{d \tilde{x}} \frac{\sin ^{2} \tilde{x}}{\tilde{x}^{2}}+\frac{\chi_{0}^{2}}{4} \frac{d}{d x} \frac{\sin ^{2} x}{x^{2}}+\frac{\chi_{0}}{2} f(x, \tilde{x})\right] .
\end{aligned}
$$

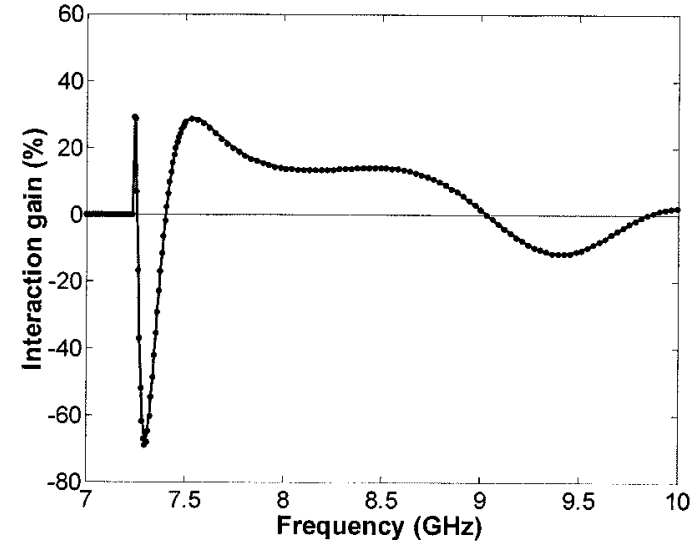

Fig. 2. Small-signal gain of an $X$-band second-harmonic waveguide FEL calculated using (14) derived from Madey's theorem (dots) and using (43) derived from the perturbation theory (solid line) at $p=0.46$.

It is worth emphasizing that the small-signal gain in second-harmonic waveguide FELs formulated in (43) is derived from a direct solution of their pendulum equation using a perturbation theory to the second order of the radiation field. Its format is very similar to that derived from Madey's theory in (14). However, they are not identical with a noticeable difference between $f(x, \tilde{x})$ and the last term in (14), as well as differences in the gain coefficient. To see this quantitatively, we consider an $X$-band second-harmonic waveguide FEL with $V_{e}=95 \mathrm{kV}$, $B_{w 0}=0.1431 T, \lambda_{w}=3.8 \mathrm{~cm}, L=11 \lambda_{w}, I=20 \mathrm{~mA}$ and $p=0.4646$. Interaction gains calculated with (14) and (43) are plotted as a function of frequency in Fig. 2. It is clearly shown that these two gain curves are in an excellent agreement. Further numerical calculations for cases with larger wiggler fields (with $p=0.5-0.8$ ) result in a similar agreement. These numerical studies suggest that Madey's theorem is indeed applicable to second-harmonic waveguide FELs even though their beam-wave interaction is much more complex than that of their conventional counterparts.

\section{CONCLUSION}

As a novel beam-wave interaction configuration to reduce the electron energy necessary for radiation at a given frequency, second-harmonic waveguide FELs have been studied in this contribution with and without Madey's theorem. In order to maximize their interaction gain at a given electron beam current, it is advantageous to operate second-harmonic waveguide FELs with a strong wiggler field. To this end, the axial electron velocity was Taylor expanded to the eighth order of the wiggler field to capture accurately the highly nonlinear dependence of electron trajectory upon the wiggler field.

$$
\begin{aligned}
\left\langle\dot{\Phi}_{2}\right\rangle_{p}= & \frac{\tilde{\Omega}^{2}}{c}\left\langle\frac{1}{\beta_{z}}\right\rangle \int_{0}^{z} d z\left\{\frac{\tilde{\Lambda}^{2}}{2}[\Delta \tilde{k} z \cos \Delta \tilde{k} z-\sin \Delta \tilde{k} z]+\frac{\Lambda^{2}}{2}[\sin (\Delta \tilde{k}-\Delta k) z+\Delta k z \cos \Delta \tilde{k} z-\sin (\Delta \tilde{k} z)]\right\} \\
& +\frac{\Omega^{2}}{c}\left\langle\frac{1}{\beta_{z}}\right\rangle \int_{0}^{z} d z\left\{\frac{\Lambda^{2}}{2}[\Delta k z \cos \Delta k z-\sin \Delta k z]+\frac{\tilde{\Lambda}^{2}}{2}[\sin (\Delta k-\Delta \tilde{k}) z+\Delta \tilde{k} z \cos \Delta k z-\sin (\Delta k z)]\right\}
\end{aligned}
$$


Madey's theorem was then assumed and used to formulate the small-signal gain and numerical examples were subsequently used to demonstrate the extended applicability of the resulted gain formula to a full parametric range of wiggler magnets. On the other hand, it was noted that Madey's theorem had not been established for second-harmonic waveguide FELs whose interaction behaviors are considerably different from that of conventional waveguide FELs. To address this, an independent gain formulation was developed by solving the pendulum equation of second-harmonic waveguide FELs with a perturbation treatment. With necessary accounts of strong wiggler fields in a number of derivation steps, the small-signal gain was again formulated, but without recourse to Madey's theorem. While this second gain formula is not analytically identical to that derived from Madey's theory, numerical examples were used to show that they agree with each other over a wide range of the wiggler field as well as other system parameters. Effectively, this validates the applicability of Madey's theorem to second-harmonic waveguide FELs.

It was shown that the choice of the wiggler field is a compromise between a large interaction gain and a significant electron energy reduction. Therefore, the implementation of second-harmonic waveguide FELs in future designs of compact waveguide FEL systems is likely to be influenced by specific application constraints. To minimize the electron energy and the cost of the overall FEL system, other electron energy reduction techniques need to be considered in parallel and assessed against each other. Examples of these techniques include a similar but different second-harmonic scheme based on a transverse beam-wave interaction achieved in a rectangular waveguide [21], a radio-frequency modulation based harmonic generation scheme [7] and a nonlinear harmonic generation scheme [22]. It is conceivable that the individual development of these novel harmonic generation techniques will collectively lead to an economically important reduction in the electron energy and hence in the capital cost of compact waveguide FELs.

\section{REFERENCES}

[1] J. F. Schmerge, W. J. W. Lewellen, Y. C. Huang, J. Feinstein, and R. H. Pantell, "The free-electron laser as a laboratory instrument," IEEE J. Quantum Electron., vol. 31, pp. 1166-1171, 1995.

[2] G. P. Gallerano, A. Doria, E. Giovenale, and A. Renieri, "Compact free electron lasers: From Cerenkov to waveguide free electron lasers," Infrared Phys. Technol., vol. 40, pp. 161-174, 1999.

[3] A. Doria, G. P. Gallerano, and A. Renieri, "Kinematic and dynamic properties of a waveguide FEL," Opt. Commun., vol. 80, pp. 417-424, 1991.

[4] G. Kong, J. Lucas, and R. A. Stuart, "Low voltage ubitrons for industrial purposes," Nucl. Instrum. Methods A, vol. 304, pp. 238-242, 1991.

[5] Y. Y. Kawamura, B. C. Lee, M. Kawai, and K. Toyoda, "Various characteristics of a waveguide mode free-electron laser using a long-pulse relativistic electron-beam," Phys. Rev. E, vol. 47, pp. 4399-4404, 1993.

[6] F. Ciocci, R. Bartolini, A. Doria, G. P. Gallerano, E. Giovenale, M. F. Kimmitt, G. Messina, and A. Renieri, "Operation of a compact freeelectron laser in the millimeter-wave region with a bunched electronbeam," Phys. Rev. Lett., vol. 70, pp. 928-931, 1993.

[7] A. Doria, G. P. Gallerano, E. Giovenale, S. Letardi, G. Messina, and C. Ronsivalle, "Enhancement of coherent emission by energy-phase correlation in a bunched electron beam," Phys. Rev. Lett., vol. 80, pp. 2841-2844, 1998.

[8] D. E. Pershing, R. D. Seeley, R. H. Jackson, and H. P. Freund, “Amplifier performance of the NRL ubitron," Nucl. Instrum. Methods A, vol. 358, pp. 104-107, 1995.

[9] R. Drori, E. Jerby, and A. Shahadi, "Free-electron maser oscillator experiment in the UHF regime," Nucl. Instrum. Methods A, vol. 358, pp. $151-154,1995$.

[10] M. G. Kong, "Energy spread analysis of a waveguide optical klystron," Opt. Commun., vol. 132, pp. 464-468, 1996.

[11] E. Jerby, "Traveling-wave free-electron laser," Phys. Rev. A, vol. 44, pp. 703-715, 1991

[12] D. E. Pershing, R. H. Jackson, H. P. Freund, M. Blank, K. Nguyen, and J. M. Taccetti, "Design of a slow-wave ubitron," Nucl. Instrum. Methods A, vol. 375, pp. 230-232, 1996.

[13] J. M. Taccetti, R. H. Jackson, H. P. Freund, D. E. Pershing, and M. Blank, "A CHI wiggler ubitron amplifier experiment: Wiggler characterization," Nucl. Instrum. Methods A, vol. 375, pp. 496-499, 1996.

[14] M. G. Kong, "A low voltage waveguide free electron laser based on a longitudinal interaction mechanism," Opt. Commun., vol. 141, pp. 48-52, 1997.

[15] X. Zhong and M. G. Kong, "Effects on nonresonant electron velocity components in a second harmonic waveguide free electron laser," Opt. Commun., vol. 168, pp. 375-381, 1999.

[16] G. Dattoli and A. Renieri, "Experimental and theoretical aspects of the frr-electron laser"," in Laser Handbook, M. L. Stitch and M. Bass, Eds. Amsterdam, The Netherlands, North-Holland, 1985, vol. 4, pp. $1-141$.

[17] J. H. Booske, B. D. McVey, and T. M. Antonsen Jr., "Stability and confinement of nonrelativistic sheet electron beams with periodic cusped magnetic focusing," J. Appl. Phys., vol. 73, pp. 4140-4155, 1993.

[18] A. Sneh and E. Jerby, "Coaxially fed folded foil electromagnetic wiggler," Nucl. Instrum. Methods A, vol. 285, pp. 294-298, 1989.

[19] J. M. J. Madey, "Relationship between mean radiated energy, mean squared radiated energy and spontaneous power spectrum in a power series expansion of the equation of motion in a free-electron laser," Nuovo Cimento B, vol. 50, pp. 64-88, 1979.

[20] W. B. Colson, "Free-Electron Laser Theory," Ph. D. dissertation, Stanford University, Stanford, CA, 1977.

[21] H. Bluem, R. H. Jackson, H. P. Freund, D. E. Pershing, and V. L. Granatstein, "Demonstration of a new free-electron laser harmonic interaction," Phys. Rev. Lett., vol. 67, pp. 824-827, 1991.

[22] H. P. Fruend, S. G. Biedron, and S. V. Milton, "Nonlinear harmonic generation in free-electron lasers," IEEE J. Quantum Electron., vol. 36, pp. 275-281, 2000

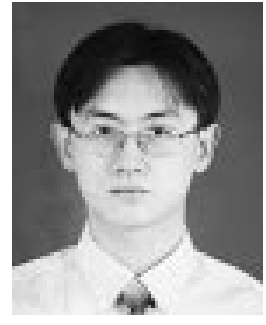

(U.K.)
Xiehe Zhong (S'01) was born in Shenzhen, China, in 1975. He received the B.Sc. degree in electronic engineering from Shenzhen University, China, and the M.Sc. degree in telecommunications engineering from Liverpool University, U.K., in 1997 and 1998, respectively. He is working toward the Ph.D. degree at Loughborough University, U.K.

His present research interest includes compact waveguide free electron lasers and optical communications systems.

Mr. Zhong is an Associate Member of the IEE

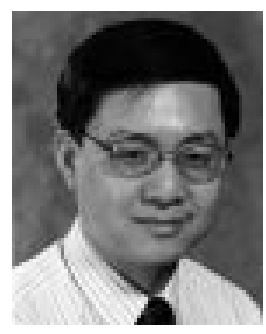

Michael G. Kong (M'94-SM'98) received the B.Sc. and M.Sc. degrees, in electronics engineering, from Zhejiang University, China, and the Ph.D. degree in electrical engineering from Liverpool University, U.K., in 1984, 1987, and 1992, respectively.

He has been a Senior Lecturer at Loughborough University, U.K. since 1999, and prior to that he was a Lecturer at Liverpool University, U.K. between 1995 and 1999. He is a committee member of the Ion and Plasma Surface Interaction Group at the Institute of Physics, U.K. and a Chief Examiner for the Engineering Council, U.K. He has been actively involved in research on compact waveguide free electron lasers, nonthermal atmospheric gas discharges and power systems and devices. In these subject areas, he has authored 80 published papers in scientific journals and peer-reviewed conference proceedings. 